\title{
NUEVAS INCURSIONES EN EL VOCABULARIO DE TEODORO PRISCIANO. FORMAS VERBALES “TÉCNICAS” Y TARDÍAS
}

\author{
Matilde Conde Salazar \\ Instituto de Filología, CSIC
}

This article analyse the verbs which were testified since the postclassic and late periods and were used by Theodorus Priscianus, to determine their contributions to late latin lexicon. It revises the chronology, specially the innovations, philological processes (prefixation, sufixation or composition), presence in relevant works of others fields (christian, grammatical, legislative) and distribution in semantic fields (pathology, therapeutic or pharmacology).

\section{Introducción}

El propósito del presente trabajo es analizar algunas parcelas del vocabulario de los tratados médicos latinos de los siglos IV y $\mathrm{V}$, en tanto en cuanto suponen una importante aportación al acervo léxico de toda la latinidad tardía.

Estos autores y obras, relegados al olvido durante un período de importantes avances de la Filología y la Crítica Textual, están siendo objeto de revisión desde hace ya algunos años y los diferentes estudios que se les han dedicado han llevado a conclusiones importantes sobre a la calidad de los mismos, tanto respecto a su contenido, que supera el de ser meras compilaciones de los grandes creadores de la ciencia médica en la Grecia clásica, como respecto a su lengua, que no se distingue especialmente por los vulgarismos ${ }^{1}$. Todo ello convierte a estos médicos en auténticos profe-

1 Cf. D. R. Langslow, «Latin Technical Languages: Synonyms and Greek Words in Latin Medical Terminology», Transactions of the Philological Society 87, 1989, pp. 33-53, esp. 
sionales que elaboran manuales técnicos escritos en latín donde manifiestan ciertas preocupaciones literarias e, incluso, se atisba una búsqueda de la belleza formal en sus composiciones.

Abordamos, de forma concreta, la lengua de Teodoro Prisciano ${ }^{2}$, médico africano discípulo de Vindiciano (magister meus ... qui nunc orbis totius Vindicianus celebratur, Physica 3). La producción latina que conservamos, conocida como Euporiston libri tres, es una traducción hecha por el autor, según propia confesión ${ }^{3}$, del original que él mismo había redactado en principio en griego. La primera y única edición crítica existente hasta el momento, se la debemos a V. Rose ${ }^{4}$, aunque fue revisada en profundidad por Sundelin, en sucesivos trabajos desde 1895 hasta $1934^{5}$.

Las incursiones que en otras ocasiones hemos realizado en el vocabulario de este autor ${ }^{6}$ nos permiten descubrir en él una clara conciencia de estar gestando tratados especializados de la ciencia médica, lo que no le exime de estar profundamente influido por la lengua de la época en que escribe.

Con frecuencia algunos de los términos técnicos que emplea no son exclusivos de la medicina, sino que los comparte con otras ciencias ${ }^{7}$, lo que

p.33; «Some historical developments in the terminology and style of Latin medical writings», Tradición e Innovación de la Medicina latina de la antigüedad y de la Alta Edad Media, Santiago de Compostela 1994, pp. 233 ss.; P. Mogliorini, «Eufemismi e varianti lessicali in Teodoro Prisciano», Anazetesis. Quaderni di ricercha 6-7, 1982, pp. 24-35.

2 Una bibliografia bastante completa sobre el autor y su obra se puede encontrar en A. Önnefors, «Das medizinische Latein bis Cassius Felix», ANRW 37,2, Berlín 1993, pp. 227-391, esp. pp. 288-299 y 387-392.

3 Nuper me collegae Olympii exhortatione prouocatum nonnullos confecisse praesentaneae libellos medicinae uel mediocris fama retinet, sed graeco stylo quoniam medendi industriam sermone claro haec natio-publicauit ... (Euporista Faenomenon 1). Citaré por abreviatura del nombre del libro y capítulo de la edición de Rose.

4 V. Rose, Theodori Prisciani Euporiston libri III, Leipzig, 1894.

5 Cf. A. Önnefors, ob. cit., pp. 288-300 donde se analizan detenidamente algunas de sus aportaciones.

6 Cf. M. Conde - A.Moreno, «Estudios del léxico tardío de los tratados latinos africanos de los siglos IV y V», en Tradición e Innovación ... pp. 241-251; M. Conde - M ${ }^{\mathrm{a}}$. J. López de Ayala, «Recursos literarios en la obra de Teodoro Prisciano», en Les textes médicaux comme littérature. VI Colloque International sur les textes médicaux latins (en prensa).

7 Cf. I. Mazzini, «Il lessico medico latino antico: caratteri e strumenti della sua differenziazione», en Le latin médical. La constitution d'un langage scientifique. Realités et langage de la médecine dans le monde romain, ed. G. Sabbah, Saint Étienne, 1991, 
es de suma importancia para el estudio del latín tardío. La evolución del lenguaje técnico se dirige hacia una nominalización ${ }^{8}$ que supone la decadencia en el uso y creación de formas verbales; se tiende a explicar una determinada acción o proceso mediante su denominación a base de un término concreto o, en el mejor de los casos, por una lexía compuesta ${ }^{9}$. Este fenómeno incide también, inevitablemente, en el estudio de las características del latín tardío.

A pesar de estos hechos constatados, observamos que los autores de tratados técnicos emplean y crean formas verbales cuyo uso, en muchos casos, queda restringido a obras de esas características e incluso, concretamente en nuestro caso, a tratados de medicina. Es en este sentido en el que, con las reservas lógicas, hablamos de tecnicismos. El hecho nos sugiere que puede ser interesante para analizar el material $\mathrm{y}$, a la vista de los resultados obtenidos, no sólo valorar la importancia de esta creación, sino también establecer el grado de innovación que supone, así como su integración dentro de la lengua latina tardía.

Por esta razón, el estudio se ha restringido al campo verbal en este autor, con el fin de estudiar las formas documentadas a partir del s. I y, sobre todo, las de los siglos II y III en adelante, aunque también se contemplan algunos testimonios anteriores, pero de escasa incidencia en los mismos y con marcado carácter técnico. Todo ello con especial atención a los factores morfológicos, al ámbito, o los ámbitos, en los que se generaliza su uso, grado de coincidencia entre campos científicos diferentes y, dentro del de la medicina, el campo léxico o semántico al que pertenece.

La creación de nuevas formas se realiza fundamentalmente mediante mecanismos comunes al resto del vocabulario ${ }^{10}$ : calcos, prefijación, adición de algún tipo de infijo o sufijo para reforzar el significado de la forma simple ${ }^{11}$, y composición.

pp. 175-185; L.Callebat, «Langages techniques et langue commune», en Latin vulgaire-latin tardif II. Actes du IIème. colloque international sur le latin vulgaire et tardif, Tübingen, 1990, pp. 45-46; D. R. Langslow, «Some historical developments...», p. 231-236.

8 Cf. D. R. Langslow, «Some historical developments ...», p. 236 ss.

9 Según la terminología de B. Pottier "lexía" es la unidad funcional significativa del habla, frente a morfema y palabra. Cf. J. Dubois y otros, Diccionario de Lingüistica, Madrid, 1979 , p. 387.

10 J. Untermann, «Etymologie und Terminus technicus», MS 1978, p. 5; R. D. Langslow, «Some Historical Developments ...», pp. 236-237.

11 Aunque este matiz deja de percibirse hasta el punto de sustituir semánticamente al verbo simple. 


\section{Estudio léxico}

Se ha dividido el material en una serie de períodos en función de la primera documentación escrita disponible hasta el momento y, dentro de cada apartado, se atiende a su característica de simple, prefijado, sufijado o compuesto. El esquema resultante es el siguiente:

1. Verbos documentados desde época arcaica o clásica bien de forma esporádica bien con un marcado carácter técnico. ${ }^{12}$

Simples: coagulo (Var.), glut(t)io (P1.), malaxo (Var.), manifesto (Ou.), tussio (P1.)

Prefijado: denigro (Var.), desicco (P1.), distermino (Cic.), enutrio (Ou.), exhalo (Pacuu.), inquoquo (Verg.), inunguo (Cato), progenero (Var.), reformo (Ou.), superaddo (Verg.).

Sufijados: fumigo (Var.), acesco (Hor.)

Prefijo + Sufijo: emadesco (Ou.), expauesco (Hor.), incandesco (Catull.)

2. Verbos documentados desde autores técnicos del siglo $\mathrm{I}^{13}$ :

Simples: bullio (Vitr.), colo (Colum.), cribro (Plin.), germino (Colum.), glutino (Cels.), mundo (Colum.), scariph(ic)o (Plin.)

Prefijados: adimpleo (Colum.), attestor (Plin.), discoquo (Plin.), exsuc(c)o (Vitr.), inquieto (Colum.), interseco (Vitr.), percuro (Plin.), pertraho (Plin.), praeualeo (Plin.), recorrigo (Sen.), recuro (Plin.), supernato (Sen.)

Sufijados: febricito (Cels.), pigresco (Plin.)

Prefijo + Sufijo: emarcesco (Plin.), infrigesco (Cels.)

Compuesto: pinguefacio (Plin.)

3. Verbos documentados desde los siglos II-III (generalmente en autores cristianos):

Simples: minoro (Tert.), candido (Tert.)

Prefijados: conforto (Vet.), deuio (Vet.), delauo (Porph.), indulco (Vet.), obuio

(Vet.), permundo (Tert.), praelauo (Apul.)

Sufijados: baptizo (Tert.)

12 Entre paréntesis se reseña el autor que trasmite la primera documentación escrita del verbo. Se aclarará cuando no corresponda a la abreviatura del Thesaurus Linguae Latinae (Th.L.L.): Var.= Varro.

13 Cf. el interesante estudio que sobre términos técnicos médicos en Séneca realizó Paola Mogliorini, «Osservazioni sulla lingua della medicina in Seneca», en Munus amicitiae. Scritti in memoria di Alessandro Ronconi, Florencia, 1988, pp. 22-56. 
4. Verbos documentados desde los siglos IV-V.

4.1. en autores no estrictamente técnicos:

Prefijados: cataplasmo (Vulg.), perneco (Aug.)

Compuestos: phlebotomo (Aug.)

4.2. en tratados latinos tardíos de veterinaria o medicina:

Simples: cribello (Pall.), elixo (Apic.), fomento (Veg.)

Prefijados: praeuaporo (Cass. Fel.), superadiicio (Apic.)

Sufijados: rheumatizo (Cass. Fel.), infrigido (Veg.)

Prefijo + Sufijo: apophlegmatizo (Cass. Fel.)

5. Verbos documentados por primera vez en Teodoro Prisciano y con incidencias posteriores

Simples: masso

Prefijados: praemundo

Compuestos: ignefacio

\section{Hapax de Teodoro Prisciano}

Simples: sapono, uesico

Prefijados: defugo, praefomento, redamno, superadhibeo

Sufijados: cadesco

\section{Estratificación cronológica del vocabulario}

a) Reseñamos, en primer lugar, el empleo de una serie de formas verbales que, aunque documentadas desde época antigua, adquieren una mayor frecuencia de uso en un período más tardío: coagulo, distermino, enutrio, expauesco, fumigo, incandesco o se emplean siempre con un sentido muy técnico: acesco, denigro, emadesco, glut(t)io, malaxo.

b) Existe un importante empleo de formas verbales documentadas a partir del siglo I, no necesariamente desde el tratado médico de Cornelio Celso, sino en alguno de los tratados técnicos que empiezan a proliferar en esta época debidos a Columela (adimpleo, colo, cribro, germino, mundo), Plinio (attestor, cribro, discoquo, emarcesco, pertraho, pinguefacio, scaripho), Vitruvio (bullio, interseco) y en Séneca ${ }^{14}$ (recorrigo, superna-

14 La obra de Séneca, y de forma especial las Epistulae morales ad Lucilium, ofrecen continuas referencias al entorno de la medicina que ya han sido objeto de reflexión por parte de algunos estudiosos, cf. bibliografía en M. Conde, «El médico en las epístolas de 
to). Muchas se documentan en más de uno (febricito, colo, denigro, distermino etc).

b) Digno de mención es también el elevado empleo de formas cuya primera manifestación nos ha llegado a través de autores cristianos como son las recogidas en el apartado 3. del esquema, a excepción de los derivados de lauo: delauo, que nos facilita Porfirión y praelauo, documentado en Apuleyo.

c) El mayor número de nuevas creaciones corresponde sin embargo a una época más tardía y se reparte entre el final del siglo IV y el siglo $\mathrm{V}$. Algunas, reunidas en el apartado 4.1 del esquema, están documentadas, además, en autores no relacionados con la medicina. Éstas son cataplasmo, en Vulgata; perneco y phlebotomo en Agustín. Otras, que componen el apartado 4.2 y son de esta misma época, aparecen sólo en tratados técnicos, en herbarios o en tratados de medicina o veterinaria, así: cribello, elixo ${ }^{15} \mathrm{y}$ superadiicio en Apicio (culinario) y Paladio (agricultura), apophlegmatizo empleado más adelante por el traductor de Oribasio; praeuaporo y rheumatizo en Casio Félix. Dos términos del apartado 5 del esquema se documentan en Teodoro Prisciano y en época posterior: masso en una lectura dudosa de Agustín y en Glosarios; praemundo en las Nouellae Iustiniani ${ }^{16}$, ignefacere en Dioscórides. Por último reseñamos las formas cadesco, defugo, praefomento, redamno, saponata, superadhibeo y uesico que consideramos hapax por no haber encontrado ninguna otra documentación excepto la de Teodoro Prisciano.

\section{Características morfológicas}

Predominan las formas con prefijación que corroboran los resultados del análisis realizado en otro trabajo sobre los sustantivos ${ }^{17}$. Existe una

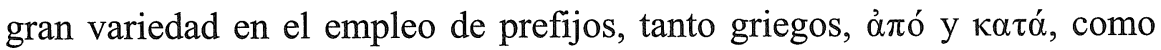

Séneca y Petrarca», en Séneca dos mil años después. Actas del Congreso Internacional Conmemorativo del Bimilenario de su Nacimiento, ed. M. Rodríguez Pantoja, Córdoba, 1997, pp. 637-644, esp. pp. 638-639.

15 Que en los comentarios a Lucano se explica como mollire ... quod uulgo elixare dicunt (Comment. Luc. 6,114).

16 Cf. tb. A. Souter, Glossary of Later Latin to 600 A.D., Oxford 1957 (=1949), s.u.

17 Cf. M. Conde - A. Moreno, ob. cit., p. 245. El predominio de la prefijación en sustativos en -tio / -sio reflejaba una mayor prefijación en la base verbal. 
latinos: ad-, co-, de-, dis-, e-lex-, in-, inter, ob-, per-, prae-, re-, super-; el prefijo latino de mayor incidencia in- (inungo, incandesco, incoquo, indulco, infrigesco, infrigido, inquieto, inunguo) seguido de de- (defugo, delauo, denigro, desicco, deuio), e- / ex-(emadesco, emarcesco, enutrio, exhalo, expauesco, exsucco), prae- (praelauo, praemundo, praeuaporo, praefomento) y super- (superaddo, superadhibeo, superadiicio, supernato).

Determinadas formas simples se utilizan como tales $\mathrm{y}$, a su vez, sirven de base léxica para la derivación con diferentes prefijos (mundo: permundo y praemundo; fomento: praefomento), aunque no todas han sido recogidas en el presente estudio por ser formas verbales clásicas: adhibeo, corrigo, curo, fugo, lauo, mundo, uaporo, unguo.

Los sufijos que se emplean en la formación de verbos ofrecen una importante variedad si se tiene en cuenta la relación entre el número de formas (12) y los distintos tipos registrados (4); no faltan algunos de claro incremento en latín tardío como el griego -1 $\zeta \omega$ (apoflegmatizo, baptizo, rheumatizo) y, sobre todo, -sco (acesco, cadesco, emadesco, expauesco, incandesco, infrigesco). También se usan -igo (fumigo) y -cito (febricito).

El procedimiento con menor incidencia es el de la composición a base de dos términos simples: phlebotomo (composición que ya existía en el sustantivo del que procede), ignefacio, pinguefacio.

Finalmente, son más numerosos los verbos que pertenecen a la primera conjugación, hasta el punto de que se prefieren formas como delauo o exsuco frente a las de otra conjugación como deluo o exsugo, aunque no faltan ejemplos tardíos de otras conjugaciones como superadiicio, que se usa en combinación con el más clásico superaddo.

\section{Procedencia del vocabulario}

El predominio de la prefijación conduce a una primera hipótesis acerca de que los nuevos verbos se hayan forjado a partir de otros simples ya existentes, algunos de ellos utilizados también por Teodoro Prisciano (lauo: Faen. 5, unguo: Faen.5 etc.) y otros no (adiicio). No faltan, sin embargo, formas denominativas (coagulo, fomento, fumigo, colo, cribro, exsuco, febricito) o adjetivales (denigro, candido, inquieto, minoro).

Los préstamos griegos son más frecuentes a partir del siglo II y, sobre todo, del IV (baptizo, cataplasmo, phlebotomo, apophlegmatizo, rheumatizo, masso). La integración de dichos préstamos es variable en función 
del momento y el colectivo que acuña el préstamo. En un porcentaje elevado se pueden considerar tecnicismos de una misma especialidad científica (medicina, veterinaria, farmacología, etc.).

\section{Confluencia con otros autores u obras técnicas}

a) Con tratados de medicina o veterinaria:

Se comprueba que el mayor índice de coincidencia en el empleo de estos verbos se da, como es natural, entre Teodoro Prisciano y tratados técnicos de medicina como los de Celso o Escribonio Largo, aunque en mayor medida con los coetáneos como Celio Aureliano, Casio Félix, Marcelo de Burdeos. Es importante la confluencia con tratados sobre veterinaria (Mulomedicina Chironis o Vegecio) y recetarios (Gargilio Marcial y Apicio), con los que guardan también estrecha relación los tratados de agricultura (Catón, Varrón, Columela o Paladio): bullio en Celso ${ }^{18}$, Api cio ${ }^{19}$, Casio Félix y Marcelo de Burdeos. | febricito en Celso ${ }^{20}$, Celio Aureliano, Casio Félix, Marcelo de Burdeos. | glutino en Celso ${ }^{21}$, Vegecio ${ }^{22}$, Casio Félix, Marcelo de Burdeos. | infrigesco Celso ${ }^{23}$, Vegecio ${ }^{24}$. | colo en Columela, Escribonio Largo ${ }^{25}$, Celio Aureliano, Marcelo de Burdeos. inung $(u)_{o}$ en Varrón ${ }^{26}$, Celso ${ }^{27}$, Escribonio Largo ${ }^{28}$. | denigro en Varrón ${ }^{29}$, Mulomedicina Chironis ${ }^{30}$. | exsuco en Apicio ${ }^{31}$, Celio Aureliano y Casio Félix. | fumigo en Varrón ${ }^{32}$ y Columela ${ }^{33}$. | cribro en Gargilio Mar-

18 V 19,28 spumae argenti selibra ... cocta, donec bullire desierit

19 VIII 364 cum bene illic tres undas bullierit, leuatur.

20 I. pr. 54.3 alii et idem alias (sc. causas) post istas non febricitarent.

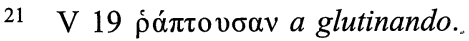

22 Mul. I 46,2, curabis clysteris collecticis id est quae glutinent.

23 V 25 cum infrixerit, catapotia ex eo fiunt.

24 Mul. II 92, 3 si amplius (sc. sanguinis) sustuleris, infrigescit.

25 134: postridie colatur liquor.

26 Ling. VI 82 oculos inunguimus.

27 VI 6,34 recte inungari potest.

2819 inungueatur.

$29 R$. I 55,7 non solum denigrare terram, sed multitudine facere sterilem.

30788 denigrare pilos.

31 Exc. 371 exsucato ipso iure.

$32 \quad R$. III 16,17 fumigans leniter eas.

33 VIII 5, 16 deinde ... surculis fumigentur. 
cial ${ }^{34}$ y Casio Félix. | cribello en Mulomedicina Chironis ${ }^{35}$, Apicio ${ }^{36}$ y Marcelo de Burdeos. | elixo en Apicio ${ }^{37}$, Casio Félix y Marcelo de Burdeos. | cataplasmo en Mulomedicina Chironis ${ }^{38}$, Celio Aureliano y Casio Félix. | malaxo en Séneca ${ }^{39}$, Casio Félix, Celio Aureliano y Marcelo de Burdeos. | progenero en Columela ${ }^{40}$ y Plinio ${ }^{41}$.

\section{b) Con autores cristianos:}

Considerable es asimismo el elenco de términos que registramos en escritores médicos y en textos cristianos, sirva de ejemplo el apartado 3. del esquema que deja patente un buen número de formas. Frente a lo que sucede entre los sustantivos, donde no se documentaba ningún préstamo presente en Vetus Latina o en Tertuliano y al mismo tiempo también en Teodoro Prisciano ${ }^{42}$, en esta ocasión hay que anotar la presencia de baptizo que, precisamente, se va a tecnificar dentro del cristianismo ${ }^{43}$, tecnificación que, sin embargo, no se refleja en otros ámbitos que lo siguen empleando en su sentido primitivo ${ }^{44}$. Otros préstamos que se documentan a partir de autores cristianos son cataplasmo ${ }^{45}$ (muy específico como tecnicismo médico), phlebotomo ${ }^{46}$, mundo ${ }^{47}$ y su compuesto permundo ${ }^{48}$, pertraho ${ }^{49}$, superadiciio ${ }^{50}$, tussio $^{51}$.

\footnotetext{
34 Pom. 2,5, in gastris uel qualis cribata terra ...

35278 bene trita et cribellata.

36 I 5 tunsa omnia et cribellata.

37 VIII 2.3 ceruum elixabis et subassabis.

38251 oleo cataplasmabis et defricito .

39 Ep. 66, 53 ut malaxandos articulos exoletis meis porrigam

40 VII 3, 12 si res exigit, ut plurimi mares progenerandi sint.

41 Nat. XI 46 fetus quonam modo progenerarent, magna ... fuit quaestio.

42 Cf. M. Conde - A. Moreno, ob. cit., p. 244.

43 Tert. Mon. 8 qui ... baptizauerit Christum.

44 Cf. Apic. IV 13 fasciculos apo baptizabis y Theod. Prisc., Faen. 66 ex hoc uulnus tamdiu baptizandum est.

45 Vulg., Is. 38,21 ut ... cataplasmarent super uulnus.

46 Aug., Serm. 21, 7 quando me emisti, nec saltem te phlebotomasti.

47 Tert., Pudic. 2, p. 223, 19 delicta mundantur, quae in fratrem ... admiserit

48 Vet. Lat., Deut. 30, 6 (cod. 100) permundabit dominus cor tuum.

49 Aug., Ep. 155,2, ne ab eis in hanc opinionem atque in peccata impia pertrahatur; Cassian. Conlat. VIII 8 dicens tertiam partem stellarum draconem illum se cum pariter pertraxisse.

50 Aug., Enarr. Ps. 70, 1, 15, perfectiorem facturus es laudem dei? est aliquid quod superadiciatur?

51 Ambr., Ep. V 26, 2, sed dum eum nimio amore abstinere gestiunt, tussire de ventre plus coepit; et laborabit adhuc, nisi ad medicamenta sua revertatur.
} 
c) Con otros campos técnicos:

Para mostrar la coincidencia en el empleo de términos entre distintos campos especializados nos ofrece un buen ejemplo adimpleo que, documentado por primera vez en Columela ${ }^{52}$, es frecuente en autores cristianos fuera de los cuales sólo se utiliza en obras de carácter técnico, pero no específicamente médicos: el juriconsulto Paulo ${ }^{53}$, el geógrafo Julio Honorio ${ }^{54}$ y el agrimensor Epifanio ${ }^{55}$, siendo Teodoro Prisciano el único médico que lo emplea. También se producen coincidencias con tratados de arquitectura (exsuco ${ }^{56}$ ), de gramática y legislativos (minoro ${ }^{57}$, praemun$\left.d o^{58}\right) \mathrm{y}$, de forma reiterada, con autores como Séneca (inquito ${ }^{59}$ ) y Apuleyo (colo ${ }^{60}$, fumigo ${ }^{61}$ ).

\section{Distribución por campos léxicos}

Si asignamos las formas estudiadas al campo léxico correspondiente, a partir de su empleo en Teodoro Prisciano, tendríamos el resultado siguiente:

\section{Patología:}

acesco 'avinagrarse': Faen. 92 flegmate supernatante frequenter acescunt cibi. apophlegmatizo 'vaciar la mucosidad': Log. 15 expedit ... apophlegmatizare in oxymeli ysopi ... decoctione.

attestor 'probar, confirmar': Faen. 17 quae profecto ex umorum colore originem causarum attestatur; | 31 plenitudinem sanguinis attestabuntur; | 67 rite beneficium attestamur; | Log. 20 releuari potius attestantur; | 35 haec eadem professio nominis attestatur; | 75 huic passioni alii collegae probasse $<$ se $>$ fuerint attestati.

52 XII 43.1, optimi generis mustum adinpleto.

53 Paul., Dig. 29.2.7541.si adimplemerit.

54 Cosmogr. geogr. p. 29 de decem fluminibus adimpletur Ganges.

55 Metrol. 4 qui apud Cyprios XVII sextarii adimpletur.

56 Vitr. 2,10: non habentes interueniorum raritates siccitatibus exsuctae solidantur.

57 Dig. 18.7.10 minorato pretio uendidit.; Don., Ter. Ad. 149 «aliquid» ... ad minorandum est; Schol. Verg. Bern. Ecl. 6,16 honorem est suum exilio minoratum esse.

58 Novell. Iust. 74, 1, 11 praemundans quidem eos alio quodam.

59 Ep. 96, 3 uesicae te dolor inquietauit; cf. P. Mogliorini, «Osservazioni ...», pp. $22-56$.

60 Flor. 6 aruum colere et aurum colare.

61 Apol. 58 suspicatus est noctu potissimum parietes fumigatos?. 
cadesco 'caer': Log. 47 stridor consequitur dentium cum praecordiorum tensione distenti uel contorti adescunt.

conforto 'confirmar': Log. 46 confortent supradictarum causarum sanitatem fuisse plenam capiti reformatam.

coagulo 'coagular': Log. 59 dabo .. antidota ...quae ... coagulare ualeant flegmata.

deuio 'apartarse'; Log. 6 quantum in contrariam partem natura deuiauerit.

distermino (1) 'diferenciar, distinguir': Log. 32 Satyriasis gonorroea uel priapismus, quibus similis est sub inmoderata patratione molestia, his accidentibus disterminantur; | 49 simili mentis confusione alienantur, quantitate causae et magnitudine accidentium sola disterminantur; 77 nonnulla similia sunt accidentia, sed coloris deformatione sola disterminantur.

emarcesco 'ajarse': Log. 54 si loca emarcuerint diu hoc morbo uexata.

expauesco 'temblar': Log. 50 sonitus suarum aurium expauescant.

glutino 'cicatrizar': Faen. 63 quod glutinare et curare membra ualeat uulnerata; | ibid. continuo etenim et glutinat et colligat; | 85 purgando et glutinando; Log. 66 constringere et glutinare poterunt.

infrigesco 'enfriarse': Log. 94 flegmatis infestatione stomachus infrixerit.

infrigido 'enfriar': Faen. 33 pusca frigida frequenter infrigidetur; 72 infrigidatae enim partes; | $\log .7$ qua infrigidare corpora ardentia uideantur; | 39 infrigidare sufficiant; | 63 ex oleo roseo infrigidato.

inquieto 'inquietar, doler': Faen. 60 si ... uesiculis fuerint inquietati; | Log. 41 si uero de frigore caput fuerit inquietatum; | 57 omne corpus inquietat; | 59 si fauces inquietauerit; | 65 si etiam tussicula coeperint inquietari; | 93 calore nimio inquietatur; | Gyn. 2 grauis dolor inquietat

febricito 'tener fiebre': Faen. 72 febricitantibus emergens curatur; | Log. 3 febricitantibus itaque generaliter omnibus unum magnum remedium; | Log. 10 febricitantibus omnes iniectiones meningae sociari.

germino 'germinar': Faen. 2 plura germinant salutis officia.

manifesto 'hacer patente, manifestar': Log. 6 est autem urina naturaliter quae corporis quietem et sanitatem manifestet; $\mid \log$. 20: Peripleumonicos haec accidentia manifestant; $\mid \log$. 35: sudor uiscosus et frigidus perseuerans interemptis uiribus manifestat.

minoro 'disminuir, aminorar': Faen. 29 minoratae scrofae penitus curabantur; | Log. 52 causa minorata non fuerit; | 55 repentina contractione minorata; | Gyn. 8 minoratis uero supradictis accidentibus.

perneco 'matar': Log. 37 sub angusto tempore homines pernecauit. pigresco 'coger pereza': Log. 26 pigrescunt ad omnia;

praeualeo 'prevalecer': Log. 60 constrictionis molestia saepe praeualeat; progenero 'engendrar': Faen. 16 fructus cum raritate progenerat.

recorrigo 'corregir': Faen. 39 uitium recorrigetur;

redamno 'revocar, rechazar': Log. 51 suam sententiam redamnantes.

reformo 'restablecer, mejorar': Faen. 38 oculorum aciem reformauit; | Log. 45 secundum naturam sanitatem reformando securus confidat commotiones minime profecturas; | 46 sanitatem fuisse plenam capiti reformata. 
rheumatizo 'tener catarro': Faen. 35 si oculi reumatizauerint; | 53 faucibus indignatis reumatizauerit; | Log. 58 conueniet interea per initia reumatizanti diuturnam quietem indici; | 68 fit ex capite reumatizante frequenter; | 103 semper enim inportunae uigiliae corpora reumatizantia fatigarunt; | 109 indignantibus et reumatizantibus renibus.

tussio 'toser': Log. 20 tussiunt frequenter, sputa sanguinea et sordida egerunt.

\section{Terapéutica:}

\section{Asepsia}

adimpleo 'llenar': Faen. 17 si ..cauernas adimpleas; | Log. 107 uentrem satis adimpleuerit.

baptizo 'lavar': Faen. 66 ex hoc uulnus tamdiu baptizandum est.

delauo 'lavar': Faen. 84 frequentius delauari, | Ibid, is aeger se cum haemorroides pateretur frequentius delauarit; | 94 non delauatis prioribus.

desic(c)o 'secar': Faen. 53 continuo desiccatur; | Log. 60 omnia loca ... posse facilius desiccari; | 62: corpus omne iam reumatismo plene possessum aliquatenus ualeam desiccare; | ibid. loca desiccare, | 107 adhibenda cataplasmata desiccantia; | Gyn. 14 desiccari et calefieri matrix possit,

emadesco 'humedecer': Faen. 53 sub lingua teneant et quod emaduerit glutiant. mundo 'limpiar': Faen. 45 commiscebis et mundabis; | 58 cum uero mundatae fuerint et percuratae; | Log. 67 possint mundari facilius.

permundo 'limpiar completamente': Faen. 40 albas maculas permundauit; | 52 uulnera delicate permundat.

praemundo 'limpiar previamente': Faen. 32 praemundatis interea oculis. praeuaporo 'fumigar': Faen. 8 praeuaporatis locis asperges.

praelauo 'lavar de antemano': Faen. 82 cinis uino tepido praelauantibus.

\section{Medidas terapéuticas}

defugo 'hacer salir': Faen. 11 capillos locis propriis defugabis.

distermino (2) 'separar, dividir': Gyn. 12 ea tamen ratione ut haec patefactio linteolis aut aliis adiutoriis patefacientibus diutius disterminetur.

ignefacio 'abrasar, quemar': Log. 64 uirgulam auri uel argenti ignefactam, | 80 lapides molares ignefacti.

percuro 'curar completamente': Faen. 56 scabias totius faciei percurat, | 58 agrestes impetigines percurabis, | ibid. mundatae fuerint et percuratae, | 59 omnes ustiones unguendo percurat, | 60 omnes uulnerationes ex ustione uenientes facilius percurabimus, | 64 quo continuo percurari possit, | $65 \mathrm{com}$ pendiosius percurabunt, $\mid 66$ similibus adiutoriis supradictis percurabuntur, | 89 pix liquida inlinita percurat, | 90 nisi percurauerit, | Log. 46 omnia paene uetusta uitia percurantis, | 83 frequentius percuratum est, | Gyn. 3 sub una egestione possint penitus percurari. si uero minime percurari possint.

pertraho 'extraer': Log. 34 uti sane lectionibus animum ad delicias pertrahentibus, | Gyn. 25 uenientia pecora pertraxerunt. 
recuro 'corregir': Faen. 18 oleo roseo uel adipibus anserinis recuranda erunt, | Log. 110 molestias recurantes, | 113 calidis chalasticis et amycticis recurentur, | Gyn. 8 inclinationes matricis recuremus.

\section{Prevención}

obuio 'prevenir, remediar': Faen. 31 fomentatione nos conueniet obuiare, $\mid \log$. 60 nouimus etenim causis difficilibus magnis medicinae remediis obuiari, | Gyn. 5: omnibus ... stipticis obuiandum est, | 27 adiutoriis salubribus obuiamus.

\section{Aplicación de remedios}

cataplasmo 'poner una cataplasma': Faen. 22 cum oleo contrito cataplasmentur, | 33 in oxymeli coctis cataplasmetur, | 65 altiores percussus cataplasmabimus, | Faen. 71 ficis tritis incoctis cataplasmabo, | 76 trito cum melle cataplasmabimus, | ibid. ex aqua cataplasmabimus, | 77 cerussae semunciis singulis commixta cataplasmabis, | Log. 65 cataplasmandi sunt de subiectis, | 67 cataplasmandi sunt interea ex ysopi puluere, 73 stomachus alfitis cum uino cataplasmandus, | 78 cataplasmabo eos interea, | 96 foliis in uino coctis et tritis cataplasmari, | Gyn. 4 ex his cataplasmando lactis auxerunt fecunditatem.

enutrio 'alimentar, nutrir': Faen. 96 per cibos et potiones enutriantur.

exhalo 'exhalar': Faen. 9 agitabis quamdiu ueluti calefactum proprium odorem exhalet.

fomento 'aplicar fomentos': Faen. 35 saepe oculi fomentandi sunt, | 66 uulnus secundum fysicos fomentandum est, | 71 fomentari loca suadeo, | 77 ex aqua marina frigida fomentabis, | Log. 11 aqua calida, ..., fomententur, | 12 media eorum consideratis temporibus oleo dulci calido fomentanda sunt.

praefomento 'lavar antes': Faen. 66: praefomentanda sunt interea uulnera.

fumigo 'ahumar': Faen. 34 carbonibus impositis fumigandi sunt, | 46 dentes dolentes fumigando.

inungo 'untar, frotar': Faen. 37 ex suco qui manauerit inunguo cum uino commixto, | Ibid. oleo cedrino inunguere, | 38 oculos inunguimus, | 39 eodem umore inunguendi erunt, | 41 pro collyrio inunguimus, | Log. 64 murra pingui inunguendi sunt.

phlebotomo 'sangrar': Faen. 44 flebotomandus erit, | 95 flebotomandi sunt et purgandi frequentius, | Log. 77 flebotomandus est, | Gyn. 14 flebotomanda erit de talo quam maxime.

pinguefacio 'tomar cuerpo': Faen. 83 pinguefacto frequenter loca tangantur, |

Log. 91 coquo ut paulatim pinguefiat, | Gyn. 22 ut paulo pinguefiat.

scarif(ic)o 'hacer una incisión': Faen. 39 ferro scarifabis, | Log. 50 radendum est, item etiam scarifandum.

superaddo (1) 'poner sobre, aplicar': Faen. 17 superaddo purgationis genus; | 94 quinque diebus superaddendo linimenta; | Log. 17 cataplasmata chalastica cum melle de seminibus superaddo; 33 ipsis partibus durarum superaddimus fricationem manuum; 41 superaddi aliquid adiutorio debere caloris aduerteris, | 81 emplastra haec utilia superaddimus. 
superadhibeo 'aplicar sobre': Log. 44 fronti linimenta haec superadhiberi suadeo.

\section{Forma de aplicación:}

masso 'masticar': Faen. 21 massando caput purgabis, | 40 lactanti suo mater guttam ammoniaci massando et inhalando albos oculos permundauit, | 45 massare autem his frequentius conueniet.

glut(t)io 'ingerir': Faen. 53 sub lingua teneant et quod emaduerit glutiant.

\section{Farmacología:}

bullio 'hervir': Faen.12 folia tamarici in aqua bulliantur, | Gyn. 19 cum molli uapore coqui et bullire coeperint, candido 'blanquear': Faen. 6 si candidare (sc. capillos) autem uellis, colo 'filtrar': Faen. 26 hoc facilius adipes caprini uel bubuli colati efficiunt, | Faen 41 soli adipes bene colati ita sufficient, | 45 adipibus caprarum colatis tantummodo, | Log. 108 colatae ex aequo miscentur.

cribello 'cribar': Faen. 8 tundes et cribellabis, | 10 tunsa et cribellata, | 64 contundes et cribellabis.

cribro 'cribar': Faen. 7 melanthion ... teres et cribabis.

denigro 'ennegrecer, oscurecer': Faen. 40 glaucos..oculos si denigrare uolueris. discoquo 'cocer': Log. 31 maluas discoctas, | 104 holera discocta.

elixo 'hervir': Faen. 25 capparis corticem in aqua elixabis.

exsuco 'extraer el jugo': Faen. 34 in aqua calida infundes et agitando exsucabis, | Log. 91 exsucata separantur.

incandesco 'calentarse': Log. 35 quam maxime eo tempore quo aestas calidior incanduerit.

incoquo 'cocer': Log. 91 cibos incoctos excluserint.

indulco 'endulzar': Faen. 32 sucus plurimarum indulcatus decoctionum, | 96 lactis beneficio per cibos et potiones enutriantur et indulcentur.

interseco 'dividir, separar': Gyn. 17 intersecti nitri glebam.

malaxo 'reblandecer': Faen. 79 haec omnia in uino coquenda sunt et tundenda,

et malaxata.

semicoquere 'cocer a medias': Faen. 60 lenticulae semicoctae ex aqua.

saponare 'jabonar': Faen. 55 saponata lauare linimenta conueniet.

superaddo (2) 'poner sobre, aplicar; añadir': Faen. 23 superadditis tamen porco-

rum adipibus, | 68 superaddimus interea etiam de salibus quippiam, | 77 et ex

lini seminis puluere et murra ex aequo, resinae quipiam superaddito, $\mid 79$ su-

peraddita ligatura; saepissime sulphuris uiui quippiam triti superadiecimus, |

Log. 19 superaddens samsuci puluerem isdem semper adiutorium commodis-

simum, | 118 teres et superaddes aeruginis et afronitri albi uncias singulas, |

Gyn. 9 superaddens cataplasmatibus ficos tunsas.

superadiicio 'añadir': 81 sulphuris uiui quippiam triti superadiecimus.

supernato 'flotar': Log. 92 flegmate supernatante frequenter acescunt cibi, | Gyn.

19 undatio uel spuma quae supernatauerit colligitur. 
La distribución resultante de la asignación de cada término a un campo léxico no ofrece duda cuando se trata de un síntoma, de una determinada terapia o del proceso de elaboración de un fármaco, pero en otras ocasiones puede ser más problemática o discutible. En este caso hemos solucionado el problema asignando los verbos a uno u otro campo atendiendo al significado concreto que tienen en la obra de Teodoro Prisciano, así por ejemplo attestor, pertenece a la patología porque se refiere al hecho de manifestar el origen de un enfermedad; emadesco, más fácil de incluir dentro de la terapéutica porque se trata de empapar de saliva un determinado fármaco antes de ingerirlo, de hecho madefacere es el término tecnificado en los médicos latinos para referirse a la operación de humedecer ${ }^{62}$ y superadiicio expresa la adición de un ingrediente en la composición de un medicamento.

El mayor porcentaje de creación se produce en el campo de la terapéutica y posiblemente se deba a que se trata de procedimientos a los que favorece designar mediante una forma verbal. Asimismo, la aplicación terapéutica supone a veces una serie de acciones que se suceden en el tiempo y el lugar que cada una de ellas ocupa en el proceso queda perfectamente explicado por un verbo con el prefijo apropiado. De esta forma se justificaría, por ejemplo, la utilización del prefijo de anterioridad prae- (praelauo, praemundo, praefomento y praeuaporo) junto a las formas simples de la misma raiz, si bien son mayoritarios los casos en que el prefijo ha dejado de ejercer su influencia y se utilizan indistintamente formas simples y prefijadas.

Siguen en número las creaciones verbales aplicadas a la farmacología donde se engloban términos que expresan procesos con intervención de la química (denigro, candido), determinados tipos de elaboración (exsuco, lixo, semicoquo, cribro) o se explicita la adición de ingredientes (indulco, superadiicio).

Dentro de la patología, la creación verbal es más activa para la descripción de los síntomas (minoro, inquieto, febricito etc..) y muy poco para la descripción de enfermedades, parcela en la que los sustantivos tienen un puesto prioritario. Únicamente rheumatizo pertenece al campo de la patología médica.

62 Y es utilizado por Theod. Prisc. en Faen 71 sunt ... uulnerum genera quae ... ueluti malefaciendo serpunt loca uicina (cf. M. T. Santamaría, «La denominación verbal de las cualidades humorales», en Tradición e Innovación..., p. 301). 


\section{Conclusión}

Nuestro trabajo no sólo ha consistido en recopilación y análisis de formas verbales de Teodoro Prisciano, sino que además ha hecho un seguimiento de la confluencia con otros autores en el uso de los mismos y de la distribución por campos léxicos. Todo ello nos proporciona, una vez más, una serie de datos concluyentes como son:

1. En cuanto a estratificación cronológica, el núcleo más importante lo constituyen aquellas formas que se documentan desde los siglos II-III, generalmente en autores cristianos, y que no tienen carácter de tecnicismos médicos, sino que encontramos incluso tecnicismos cristianos (baptizo). Se registran también verbos de escaso contenido técnico (obuio, recorrigo) o cuya especialización es más tardía.

2. Se constata el importante y mayoritario corpus de formas clásicas que continúa en vigor.

3. Existe un considerable acervo de verbos de nueva creación en los tratados médicos latinos tardíos.

4. La mayoría de las formas verbales se especializan desde el principio como tecnicismos o expresiones propias de los médicos y así lo demuestra el tipo de fuentes que nos las han transmitido.

5. Queda también demostrado, un vez más, el empleo en las obras técnicas latinas de un acervo léxico común a todos, superpuesto a los recursos técnicos particulares de cada comunidad. El empleo de esta misma fuente por parte de los distintos especialistas produce una apariencia de intercambio de tecnicismos léxicos entre distintos tipos de tratados técnicos. Este proceso se da en todas las épocas.

En Teodoro Prisciano, y dentro del campo verbal, las nuevas creaciones creemos que se justifican como un esfuerzo del autor para realizar una obra de cierta calidad literaria ${ }^{63}$ en la que procura evitar repeticiones monótonas sin abandonar un objetivo importante del trabajo, su claridad.

63 El cuidado que Teodoro Prisciano pone en la composición de su obra es un tema interesante que atañe también al estudio del vocabulario. Algunos aspectos del mismo los hemos abordado recientemente en M. Conde- $\mathrm{M}^{\mathrm{a}}$. J. López de Ayala, «Recursos literarios...». 
Para ello se sirve de diferentes recursos lingüísticos entre los que aquí interesa recordar, por ejemplo:

a) Alternancia entre sustantivo y verbo (denigratio (hapax) / denigro; madefactio (hapax) / emadesco; uesicatio / uesico (hapax); defectum / defugo (hapax); fumigium ${ }^{64 / \text { fumigo) }}$

b) Alternancia entre formas simples y prefijadas (curo / percuro / recuro; adhibeo / superadhibeo; mundo / praemundo)

c) Alternancia léxica (curo / percuro / medeor; unguo / inunguo / adhibeo / contingo; cado / defugo / euello).

En lo que concierne a las formaciones propias de Teodoro Prisciano, hay que concluir que son pocas, ya que el autor prefiere recurrir a los cauces ya existentes en lengua latina, bien a través de sus fuentes bien, como léxico de uso en la lengua común, y que no tienen prácticamente ningún eco en escritores posteriores.

Para finalizar es importante recordar que los resultados obtenidos son parciales y deben completarse con otros del mismo tipo que den la visión completa del vocabulario de autor y que dichos resultados, incluso los negativos o poco positivos, suponen siempre nueva aportación que enriquece, sobre todo, las investigaciones en el campo del latín tardío.

64 Documentado por primera vez en Teodoro Prisciano. 